\title{
Antitoxin Reduces Botulinum Side Effects
}

\author{
ALAN B. SCOTT \\ San Francisco, USA
}

Extensive experimental application of botulinum toxin (BTX) has shown the drug to be without reported systemic toxicity in doses used in ophthalmology (Table I). However, induced vertical strabismus and/or ptosis occur in 15 to $25 \%$ of horizontal extraocular muscle injections due to diffusion from the target muscle into adjacent muscles. This is especially true of the medial rectus, where combined weakness from overflow to the supradjacent superior oblique and subjacent inferior rectus, both depressor muscles, results in hypertropia. While local side effects are usually mild and transient, they limit dosage, interfere with securing binocularity during the period of drug-induced palsy, and create vertical strabismus of two prism diopters or more at six months after injection in $1.8 \%$ of cases. In the eyelid, diffusion of the drug from the injected orbicularis muscle may involve both the levator, causing ptosis, and the superior rectus, causing vertical diplopia. This paper describes some experiments to limit or avoid these side effects using antitoxin (ATX). Timely use of antitoxin may be useful to avoid effects of erroneous BTX usage, too.

\section{Methods and Results}

We used botulism antitoxin $\mathrm{ABE}$, Connaught Laboratories, containing about 750 international units (IU) of type A antitoxin of equine origin per milliliter. By convention each IU neutralises 10,000 mouse $\mathrm{LD} / 50$ doses of type A toxin. Botulinum toxin type A is expressed in units, each unit being equal to a mouse $\mathrm{LD} / 50$ dose and corresponds to about 0.36 nanograms of the crystalline toxinhaemagglutin complex.

\section{Orbicularis ATX following orbicularis $B T X$}

To study the ability of small local doses of ATX to inhibit BTX effect, eleven blepharospasm patients were injected with ATX in the right orbicularis immediately after receiving BTX injections of equal doses to both eyes. ATX doses ranged from $1.0 \times 10^{-4}$ to $1.6 \times 10^{-3}$ IU of ATX per BTX unit. This dosage range had been previously worked out in mouse experiments. An additional eleven patients received delayed ATX injections, with intervals between BTX and ATX injections ranging from $\frac{1}{2}$ hour to 21 hours. Results were assessed by measuring the pre- and postinjection orbicularis force in both eyes. When direct force measurements were unobtainable, a subjective assessment of the difference in spasm duration and intensity between the two eyes was obtained by telephone followup. All responses were then graded on a scale of 0 to 3 , where $0=$ no ATX effect (response to BTX equal in both eyes) and $3=$ marked ATX effect (orbicularis force/spasm intensity much greater in the ATX-treated eye, indicating inhibition of BTX effect). After the effect of ATX was assessed, patients who had a reduced BTX effect in the ATX-treated eye were retreated with BTX to that eye to give them the usual relief from spasm.

Results are shown in Table II and summarised in Figure 1. As expected, increasing ATX doses resulted in increasing blockage of BTX effect. An unexpected result was that delaying the ATX injection for up to 5 hours following BTX injection resulted in increased blockage of BTX effect. Delays of 16-21 hours showed a reduced ATX effect, but did 
Table I Numbers and conditions of patients

\begin{tabular}{lcc}
\hline Condition & $\begin{array}{c}\text { Number of } \\
\text { patients }\end{array}$ & $\begin{array}{c}\text { Number of } \\
\text { treatments }\end{array}$ \\
\hline Strabismus & 3,787 & 6,685 \\
Blepharospasm & 2,865 & 8,261 \\
Hemifacial spasm & 608 & 1,281 \\
Nystagmus & 26 & 42 \\
Eyelid retraction & 12 & 34 \\
Myokymia & 12 & 20 \\
Entropion & 74 & 137 \\
Bell's palsy & 6 & 12 \\
VII nerve palsy & 17 & 39 \\
\hline
\end{tabular}

Table II Orbicularis ATX injection following orbicularis BTX injection

\begin{tabular}{ccc}
\hline $\begin{array}{c}\text { ATX dose } \\
\text { IU/BTX unit })\end{array}$ & $\begin{array}{c}\text { Delay } \\
\text { (hours) }\end{array}$ & $\begin{array}{c}\text { Effect of ATX } \\
\left(\begin{array}{l}0=\text { none } \\
3=\text { marked })\end{array}\right.\end{array}$ \\
\hline $1.0 \times 10^{-4}$ & 0 & 1 \\
$2.0 \times 10^{-4}$ & 0 & 2 \\
$4.0 \times 10^{-4}$ & 0 & 0 \\
$4.0 \times 10^{-4}$ & 0 & 3 \\
$4.0 \times 10^{-4}$ & 0 & 0 \\
$8.0 \times 10^{-4}$ & 0 & 1 \\
$8.0 \times 10^{-4}$ & 0 & 2 \\
$8.0 \times 10^{-4}$ & 2 & 2 \\
$8.0 \times 10^{-4}$ & 2 & 3 \\
$8.0 \times 10^{-4}$ & 3.5 & 3 \\
$1.6 \times 10^{-3}$ & 0 & 3 \\
$1.6 \times 10^{-3}$ & 0 & 1 \\
$1.6 \times 10^{-3}$ & 0 & 1 \\
$1.6 \times 10^{-3}$ & 0 & 2 \\
$1.6 \times 10^{-3}$ & 0.5 & 3 \\
$1.6 \times 10^{-3}$ & 1 & 3 \\
$1.6 \times 10^{-3}$ & 4 & 3 \\
$1.6 \times 10^{-3}$ & 5 & 2 \\
$1.6 \times 10^{-3}$ & 16 & 1 \\
$1.6 \times 10^{-3}$ & 18 & 1 \\
$1.6 \times 10^{-3}$ & 18 & 1 \\
$1.6 \times 10^{-3}$ & 21 & 2 \\
\hline
\end{tabular}

not eliminate entirely the effect of ATX on BTX-induced paralysis.

2. Levator ATX following orbicularis BTX Ten patients were injected in their right levators with ATX doses ranging from $4.0 \times 10^{-4}$ to $1.6 \times 10^{-3}$ IU of ATX following BTX injection of equal doses to the orbicularis of both eyes. Nine patients were injected with ATX immediately following BTX injection; one patient had a delay of 21 hours. (See Table
AVERAGE ANTITOXIN EFFECT

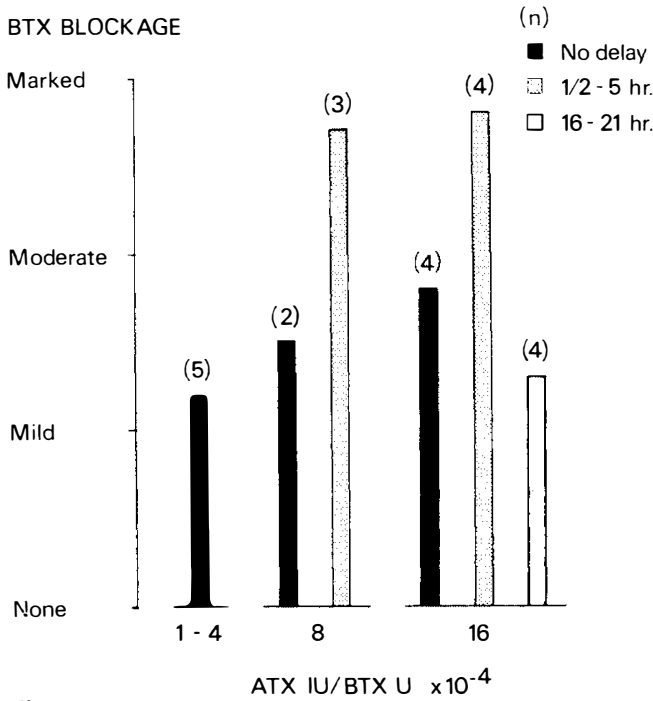

Fig. 1.

III.) Eight of the 10 patients showed ptosis in the left eye (not treated with ATX), ranging from 20 per cent to complete lid closure. Of these 8 patients, 7 had no ptosis in the ATXtreated right eye, indicating 100 per cent protection from levator paralysis, including the patient whose ATX injection was delayed by 21 hours. The other patient who had ptosis showed 50 per cent lid closure in the left eye and 25 per cent in the right eye, or a 50 per cent protection. Two patients did not get ptosis in either eye. One patient showed a slight reduction in the duration of spasm relief in the eye which received ATX, presumably by ATX diffusing forward into the orbicularis.

\section{Levator or inferior rectus ATX following horizontal rectus $B T X$ injection}

Five strabismus patients received ATX injections: to the temporal side of the levator or inferior rectus following BTX injection to the medial rectus, and vice versa for the lateral rectus. (See Table IV). Ptosis of $1 \mathrm{~mm}$ occurred in patient \#815 and slight inferior rectus weakness occurred in patient \#501. The paralysis of the target muscle was complete in all cases.

Reversal of established paralysis, even using high ATX doses, was not possible in patient \#801. 
Table III Levator ATX injection following orbicularis BTX injection

\begin{tabular}{ccc}
\hline $\begin{array}{c}A T X \text { dose } \\
\text { IU/BTX } U \\
\times 10^{-4}\end{array}$ & $\begin{array}{c}\text { Ptosis: OD } \\
\text { BTX }+ \text { ATX }\end{array}$ & $\begin{array}{c}\text { Ptosis: } \text { OS } \\
\text { BTX }\end{array}$ \\
\hline 4.0 & 0 & 0 \\
4.0 & $25 \%$ & $50 \%$ \\
8.0 & 0 & $100 \%$ \\
8.0 & 0 & $50 \%$ \\
8.0 & 0 & $50 \%$ \\
8.0 & 0 & $20 \%$ \\
8.0 & 0 & 0 \\
16.0 & 0 & $50 \%$ \\
16.0 & 0 & $25 \%$ \\
& 21-hour delay to ATX: & \\
8.0 & 0 & $75 \%$ \\
\hline
\end{tabular}

Table IV Ptosis, inferior rectus weakness after ATX

\begin{tabular}{|c|c|c|}
\hline$B T X$ & $\begin{array}{c}A T X \\
I U \times 10^{-4}\end{array}$ & $\begin{array}{c}\text { Paralytic } \\
\text { effect }\end{array}$ \\
\hline 7.5 RMR & 4.0 RIR & $\begin{array}{l}\text { RMR -4 } \\
\text { RIR -1 }\end{array}$ \\
\hline $5.0 \mathrm{LMR}$ & $\begin{array}{l}\text { 4.0 LIR } \\
\text { 4.0 Levator }\end{array}$ & $\begin{array}{l}\text { LMR -4 } \\
\text { LIR 0 } \\
\text { No ptosis }\end{array}$ \\
\hline 5.0 LMR & .16 .0 Levator & $\begin{array}{l}\mathrm{LMR}-4 \\
1 \mathrm{~mm} \text { ptosis }\end{array}$ \\
\hline 5.0 LLR & 16.0 Levator & $\begin{array}{l}\text { LLR - } 4 \\
\text { No ptosis }\end{array}$ \\
\hline \multicolumn{3}{|c|}{ 20-day delay after ATX: } \\
\hline
\end{tabular}

4. ATX to block incorrect BTX use

A 64 year old man was to be injected in the right medial rectus for esotropia with diplopia. An error was made and 5 units of BTX were given to the right lateral rectus. Thirty minutes later, $0.1 \mathrm{ml}$ (100 IU) of Lederle ATX was injected into the right lateral rectus. This provided full protection, and no change in strabismus occurred. This patient had been treated 30 years before for tetanus exposure with ATX of equine origin. No allergic response to the Lederle equine ATX ensued. Three weeks later, the left medial rectus was injected with a good paralytic and clinical effect.

\section{Discussion}

Local injections of botulism antitoxin are effective in preventing botulinum toxininduced paralysis. The dose relationships described pertain to the Connaught Botulinum Antitoxin, ABE and to the toxin of 140 units per 50 nanograms made here. Differences in potency, protein content and other factors would be very likely to alter these relationships for any other products. The Lederle ATX dose was far above the other doses of ATX used, and a much lower ATX dose would probably have been quite adequate. Short delays in ATX injection (up to 5 hours following BTX injection) result in greater blockage of BTX effects than do immediate ATX injections. We suppose that the lighter ATX molecule diffuses away sooner than does the heavy BTX. ATX injections delayed up to 21 hours are not as effective as immediate injections are, but some blockage of BTX effect still occurs, indicating that binding to nerve terminals has not yet occurred or that it is still reversible. ${ }^{1}$ This has important clinical implications in that errors of drug dilution or of drug usage may be able to be ameliorated for a number of hours after the occurrence. ATX cannot, however, reverse existing paralysis.

The small ATX doses injected into the levator or inferior rectus reduced but did not fully prevent the side effects of ptosis or vertical deviation caused by BTX strabismus injections. Higher doses should be effective as in the orbicularis. The reverse problem, diffusion of ATX into the BTX-targeted muscles which would result in a reduction of the intended effects did not seem to occur. Indeed, to the extent observable, we thought the amplitudes of angle change during paralysis of the injected eyes were greater than usual. To explain this we suppose BTX in high doses may involve also the antagonist and that ATX protects the antagonist; a beneficial effect of ATX, if so.

The occurrence of ptosis" and vertical diplopia following BTX injection at small BTX doses is a limiting factor in the treatment of a few blepharospasm and hemi-facial spasm patients. With ATX injections to the levator, however, BTX doses may be able to be increased to give a longer duration of beneficial orbicularis paralysis and to protect the levator and superior rectus in those patients who seem especially susceptible.

Strabismic children are more susceptible to 
BTX-induced ptosis and vertical strabismus than are adults. We hope that prophylactic injection of ATX to the levator and inferior rectus following BTX injection of horizontal extraocular muscles may reduce or eliminate ptosis with its risk to vision, and vertical strabismus with its barrier to binocularity.

The highest ATX dose proposed is in the range of doses proposed for use to test allergic sensitivity and is approximately one 20 millionth of the amount of the dose ordinarily proposed for use in botulinum intoxication; this latter dose gives allergic sensitivity reaction in about 2 per cent of the patients treated. ${ }^{2}$ There has been no systemic allergic reaction in this study, even in the one patient who was known to have had horse serum treatment in the past. Serum assays for antibodies to the Connaught product are under- way but not yet available. A human-derived ATX and the non-toxic large fragment of the toxin molecule to block unwanted toxin binding $^{3}$ are additional related techniques to reduce side effects and to increase efficacy which we are pursuing and which avoid the theoretical risks of immunity or sensitisation to equine-derived proteins.

\section{References}

${ }^{1}$ Simpson LL: Kinetic Studies on the Interaction between Botulinum Toxin Type A and the Cholinergic Neuromuscular Function. J Pharmacol Exp Therapeut 1980, 212: 16-21.

${ }^{2}$ Black RE and Gunn R A: Hypersensitivity Reactions Associated with Botulinal Antitoxin. Am J Med 1980, 69: 567-70.

${ }^{3}$ Krysinski EP and Sugiyama H: Purification and Some Properties of the H. Chain Subunit of Type A Botulinum Neurotoxin. Toxicon 1980, 18: 705-10. 